\title{
MINIMAL PROJECTIONS AND ABSOLUTE PROJECTION CONSTANTS FOR REGULAR POLYHEDRAL SPACES
}

\author{
BRUCE L. CHALMERS AND BORIS SHEKHTMAN ${ }^{1}$
}

\begin{abstract}
Let $V=\left[v_{1}, \ldots, v_{n}\right]$ be the $n$-dimensional space of coordinate functions on a set of points $\tilde{v} \subset \mathbf{R}^{n}$ where $\tilde{v}$ is the set of vertices of a regular convex polyhedron. In this paper the absolute projection constant of any $n$-dimensional Banach space $E$ isometrically isomorphic to $V \subset C(\tilde{v})$ is computed, examples of which are the well-known cases $E=l_{n}^{\infty}, l_{n}^{1}$.
\end{abstract}

1. Introduction. Let $\tilde{v}$ be a bounded set of points in $\mathbf{R}^{n}$. We consider the Banach space $C(\tilde{v})$ of all real-valued continuous functions on $\tilde{v}$ and a subspace $V=V(\tilde{v}) \subset$ $C(\tilde{v})$ of all homogeneous functions on $\tilde{v}$, endowed with the sup norm on $\tilde{v}$. We say that $V$ is generated by $\tilde{v}$.

So defined, $V$ forms a finite-dimensional Banach space and the relative projectional constant $\lambda(C(\tilde{v}), V)$ coincides with the absolute projectional constant $\lambda(V)$.

At this point we remark that the subspace $V$ generated by $\tilde{v}$ is isometrically isomorphic to the subspace generated by the extreme points of the convex hull of $\tilde{v}$. In this paper we will assume without loss therefore that $\tilde{v}=\operatorname{ext}(\operatorname{co}(\tilde{v}))$.

For any $t \in R^{n}, \pi_{i}(t)$ denotes the $i$ th coordinate of $t$. Under this assumption we introduce $v_{i} \in V$ by

$$
v_{i}(t)=\pi_{i}(t) \quad \forall t \in \tilde{v} .
$$

The set of functions $\left(v_{i}\right)_{i=1}^{n}$ forms a convenient basis for $V$, provided $V$ is $n$-dimensional as it is in the sequel.

In this note we construct the projection from $C(\tilde{v})$ onto $V$ with the minimal norm and compute the norm in case $\tilde{v}$ are the vertices of a regular polyhedron.

We also remark that for $n>4$ there are only three such polyhedra, and two of them generate the well-known spaces $l_{n}^{\infty}$ and $l_{n}^{1}$ for which the projectional constants and associated projections are known [3].

In $\$ 2$ we cite some known examples, in particular, the examples of the minimal projections (and their norms) onto the spaces generated by the vertices of regular two-dimensional polyhedra.

$\$ 3$ constitutes the bulk of this paper. Here we compute the projectional constants on the space generated by the tetrahedron. The space seems to be missed by all previous literature on the subject.

Received by the editors December 5, 1984.

1980 Mathematics Subject Classification. Primary 46B20; Secondary 51M20.

${ }^{1}$ Research supported in part by NSF Grant No. MCS- 8301646 . 
2. Known examples and the case $n=2$. For all $n=1,2, \ldots$ the balls of $l_{n}^{1}, l_{n}^{\infty}$ are examples of regular polyhedra in $\mathbf{R}^{n}$. Let $\tilde{v}$ denote the set of vertices $\left\{\tilde{v}_{i}\right\}_{i=1}^{k}$ where $k=2^{n}$ in the case of $l_{n}^{1}$ and $k=2 n$ in the case of $l_{n}^{\infty}$, and let $V=V(\tilde{v})=\left[v_{1}, \ldots, v_{n}\right]$ denote the associated regular polyhedral space. $v_{j} \in \mathbf{R}^{k}$ is the $j$ th coordinate function on $\tilde{v}$, i.e. $v_{j i}=v_{j}\left(\tilde{v}_{i}\right)=\tilde{v}_{i j}, 1 \leqslant i \leqslant k, 1 \leqslant j \leqslant n$. Then $P_{\min }: C(\tilde{v}) \rightarrow V$ and $\lambda(V)=$ $\left\|P_{\min }\right\|$ are known [3].

DEFINITION. If $E$ is an $n$-dimensional Banach space we say $E$ is regularizable if $E \cong$ (isometrically isomorphic to) an $n$-dimensional polyhedral space $V=V(\tilde{v}) \subset$ $C(\tilde{v})$, where $\tilde{v}$ is the set of vertices in $\mathbf{R}^{n}$ of a regular polyhedron.

EXAMPLE 1. If $\tilde{v}$ is not symmetric with respect to the origin then the $n$-dimensional Banach space $V(\tilde{v} \cup-\tilde{v}) \cong V(\tilde{v})$.

Note. In the case $n=2$ there is for every $k=2,3, \ldots$ a regular polyhedron $\pi_{k}$.

The following theorem follows for $k=2^{j}, j \in \mathbf{N}$ by comparing the Fourier projection with a result of Grünbaum [3] and for general $k=2 m$ by comparing the Fourier projection with a result of Franchetti and Votruba [2].

THEOREM 1. For $n=2$, let $\tilde{v}$ be the set of $k$ vertices of the regular polyhedron $\pi_{k}$ in $\mathbf{R}^{2}$. Then $P_{\min }: C(\tilde{v}) \rightarrow V$ is the associated Fourier projection and

$$
\lambda(V)=\left\|P_{\min }\right\|=\alpha(k)= \begin{cases}\frac{4}{k} \cot \frac{\pi}{k} & \text { if } k=4 l, \\ \frac{4}{k} \csc \frac{\pi}{k} & \text { if } k=2 m \neq 4 l,\end{cases}
$$

and $\alpha(k)=\alpha(2 k)$ if $k$ is odd.

Let $V^{k}$ denote the polyhedral space corresponding to $\pi_{k}$. Since $l_{2}^{2} \simeq V(\tilde{v})$ where $\tilde{v}$ is the unit circle in $\mathbf{R}^{2}$, we have the following expected result:

COROLlaRy. $\lambda\left(V^{k}\right) \underset{k \rightarrow \infty}{\rightarrow} \lambda\left(l_{2}^{2}\right)$.

3. The regular tetrahedron $T_{n}$. Let $\tilde{v}=\left\{\tilde{v}_{i}\right\}_{i=1}^{k}$ be the set of $k$ vertices of a regular polyhedron in $\mathbf{R}^{n}$, and let $V=V(\tilde{v})=\left[v_{1}, \ldots, v_{n}\right]$ be the associated $n$-dimensional polyhedral space. $v_{j} \in \mathbf{R}^{k}$ is the $j$ th coordinate function on $\tilde{v}$, i.e. $v_{j i}=v_{j}\left(\tilde{v}_{i}\right)=\tilde{v}_{i j}$, $1 \leqslant i \leqslant k, 1 \leqslant j \leqslant n$. The set $\tilde{v}$ is invariant with respect to the group $G$ of automorphisms induced by the regular polyhedron, yielding a subgroup of permutations of $\tilde{v}$. We say the projection $P: C(\tilde{v}) \rightarrow V$ is symmetric if $P(S f)=S(P f)$ for arbitrary $f \in C(\tilde{v}), S \in G$. We denote the set of all symmetric projections by $\mathbf{P}_{G}$.

Proposition. Let $V$ be a regular polyhedral space. Then $\lambda(V)=\inf _{P \in \mathbf{P}_{G}}\|P\|$.

Proof. Let $P: C(\tilde{v}) \rightarrow V$ be an arbitrary projection. Consider the mapping

$$
P_{G}: \frac{1}{\# G} \sum_{S \in G} S^{-1} P S \text {. }
$$

Clearly $P_{G}$ maps $C(\tilde{v})$ into $V$ and

$$
\left\|P_{G}\right\| \leqslant \frac{1}{\# G} \sum_{S \in G}\left\|S^{-1} P S\right\| \leqslant \frac{1}{\# G} \sum_{S \in G}\left\|S^{-1}\right\|\|P\|\|S\|=\|P\| .
$$


To see that $P_{G}$ is a projection onto $V$, let $y \in V$ and then

$$
P_{G}(y)=\frac{1}{\# G} \sum_{S \in G} S^{-1} P S y=\frac{1}{\# G} \sum_{S \in G} S^{-1} S y=y .
$$

Further, for $\tilde{S} \in G$,

$$
P_{G} \tilde{S}=\frac{1}{\# G} \sum_{S \in G} S^{-1} P S \tilde{S}=\frac{1}{\# G} \tilde{S} \sum_{S \in G}(S \tilde{S})^{-1} P S \tilde{S}=\tilde{S} P_{G}
$$

and so $P_{G} \in \mathbf{P}_{G}$.

REMARK 1. For $n>4$ there exist only 3 regular polyhedra, namely the balls of $l_{n}^{1}$ and $l_{n}^{\infty}$ mentioned in $\$ 2$ and the regular tetrahedron of the following theorem:

THEOREM 2. Let $\tilde{v}$ be the set of $n+1$ vertices of the regular tetrahedron $T_{n}$ in $\mathbf{R}^{n}$. Then $P_{\min }: C(\tilde{v}) \rightarrow V$ is given by

$$
\left(P_{\min } f\right)\left(\tilde{v}_{i}\right)=\frac{n}{n+1} f\left(\tilde{v}_{i}\right)-\frac{1}{n+1} \sum_{j \neq i} f\left(\tilde{v}_{j}\right), \quad i=1,2, \ldots, n+1,
$$

and $\lambda(V)=\left\|P_{\min }\right\|=2 n /(n+1)$.

Proof. Any linear operator $Q$ from $C(\tilde{v})$ onto $V$ is given by $Q f=A_{1} f, \ldots, A_{n+1} f$ where $f$ is the $(n+1)$-tuple $\left(f_{1}, \ldots, f_{n+1}\right), f_{i}=f\left(\tilde{v}_{i}\right)$, and $A_{i}$ is a fixed $(n+1)$-tuple of scalars, $i=1, \ldots, n+1$. Now, by the proposition and the symmetry of the regular tetrahedron, for $Q=P_{G} \in \mathbf{P}_{G}$, we deduce that there exist 2 fixed scalars $a$ and $b$ such that $A_{i}=(b, \ldots, b, a, b, \ldots, b)$ where $a$ occurs in the $i$ th position, $i=1, \ldots, n$ +1 . This follows since the $k$ th vertex of $T_{n}$ can be rotated to the $l$ th vertex of $T_{n}$ by the unique element of $G$ keeping the $i$ th vertex fixed, for all $i \neq k \neq l \neq i$. Next, without loss assume $\tilde{v}_{1}=(1,0, \ldots, 0)$; then it is known (and easy to check) that

$$
\tilde{v}_{i}=(-1 / n, *, \ldots, *), \quad i=2, \ldots, n+1 .
$$

Thus, $v_{1}$ is the $(n+1)$-tuple $(1,-1 / n,-1 / n, \ldots,-1 / n)$. Since $P_{G}$ is a projection, $P_{G} v_{1}=v_{1}$ whence in particular $\left(P_{G} v_{1}\right)_{1}=v_{11}$, i.e. $a-b=1$. Further, $P_{G}\left(P_{G} f\right)=$ $P_{G} f$ implies that

$$
\begin{gathered}
\left(a^{2} f_{1}+2 b a \sum_{j=2}^{n+1} f_{j}+n b^{2} f_{1}+(n-1) b^{2} \sum_{j=2}^{n+1} f_{j}, \ldots\right) \\
\equiv\left(a f_{1}+b f_{2}+\cdots+b f_{n+1}, \ldots\right)
\end{gathered}
$$

for all $f$. Hence $a^{2}+n b^{2}=a$ and $2 a+(n-1) b=1$ which yields the two solution pairs

$$
(a, b)=\left(\frac{n}{n+1},-\frac{1}{n+1}\right),\left(\frac{1}{n+1}, \frac{1}{n+1}\right)
$$

but only the first satisfies $a-b=1$. Thus $a=n /(n+1), b=-1 /(n+1)$. From the symmetry of all the $\left(P_{G} f\right)_{i}, i=1, \ldots, n+1$, we conclude that

$$
\begin{aligned}
\left\|P_{G}\right\| & =\max _{\|f\|_{\infty}=1}\left|\left(P_{G} f\right)_{1}\right|=\max _{\|f\|_{\infty}=1}\left|b f_{1}+a f_{2}+\cdots+a f_{n+1}\right| \\
& =\frac{n}{n+1}+\frac{n}{n+1}=\frac{2 n}{n+1} .
\end{aligned}
$$


Note 1. For $n>2, V(\tilde{v} \cup-\tilde{v})$ is not an unconditional (and therefore, of course, a nonsymmetric) $n$-dimensional Banach space.

REMARK 2 . Since $\tilde{v}$ contains only $n+1$ points, the best approximation operator $B$ from $C(\tilde{v})$ onto $V(\tilde{v})=V$ is linear and, in fact, $B=P_{\min }$.

REMARK 3. If $n=3$ there are 2 regular convex polyhedra other than $l_{n}^{1}, l_{n}^{\infty}, T_{n}$ and if $n=4$ there are 3 such other. These 5 examples can also be calculated analogously as in Theorem 2. In the case $n=3$, for the icosahedron $\lambda(V)=(1+\sqrt{5}) / 2$ and for the dodecahedron $\lambda(V)=.3(3+\sqrt{5})$. In the case $n=4$, for the polyhedron with 24 vertices $\lambda(V)=5 / 3$, for the polyhedron with 120 vertices $\lambda(V)=(6 \sqrt{5}+11) / 15$, and for the polyhedron with 600 vertices $\lambda(V)=(9 \sqrt{5}+22) / 25$.

For example, in the case of the dodecahedron $(n=3)$, we follow the procedure in the proof of Theorem 2 and using the symmetry (see [1]) of the dodecahedron we have $P_{G} f=\left(A_{1} f, \ldots, A_{20} f\right)$ where, with an appropriate ordering of the vertices, we have $A_{1}=(a, b, b, b, c, c, c, c, c, c, d, d, d, d, d, d, e, e, e, g)$, etc. Then using $P_{G}\left(P_{G} f\right)=P_{G} f$, we are led to six equations in the six unknowns, $a, b, c, d, e, g$. One of the solutions is obtained by taking $a=-g, b=-e$, and $c=-d$, yielding the three equations

$$
2 a^{2}+6 b^{2}+12 c^{2}=a, \quad 4 a+8 c=1, \quad 2 b^{2}-2 c^{2}+4 a c=c
$$

with solution $a=3 / 20, b=\sqrt{5} / 20, c=1 / 20$. Then

$$
\left\|P_{G}\right\|=\max _{\|f\|_{\infty}=1}\left|\left(P_{G} f\right)_{1}\right|=\max _{\|f\|_{\infty}=1}\left|A_{1} f\right|=2 a+6 b+12 c=.3(3+\sqrt{5}) .
$$

But also the solution $a=1 / 5, b=(1+\sqrt{5}) / 20, c=1 / 10, d=0, e=(1-\sqrt{5}) / 20$, $g=-1 / 10$ yields another minimal projection so that $P_{\min }$ is not unique.

REMARK 4. In fact the procedure of Theorem 2 can also be used to reobtain $P_{\min }$ and $\lambda(V)$ in the cases of the balls of $l_{n}^{1}$ (trivially $P_{\min }$ is a norm 1-interpolating projection on the vertices on the $n$ positive coordinate axes) and $l_{n}^{\infty}$.

ACKNOWLedgment. The authors are grateful to Professor L. Harper for his guidance in the world of polyhedra and to the referees for their helpful suggestions.

\section{REFERENCES}

1. H. S. Coxeter, Regular polytopes, Macmillan, New York, 1963.

2. C. Franchetti and G. Votruba, Perimeter, Macphail number and projection constant in Minkowski planes, Boll. Un. Mat. Ital. B(6) 13 (1976), 560-573.

3. B. Grünbaum, Projection constants, Trans. Amer. Math. Soc. 95 (1960), 451-465.

Department of Mathematics, University of California, Riverside, California 92521 